\title{
Observation of $7 p^{2} \mathbf{P}_{3 / 2} \rightarrow 7 d^{2} \mathbf{D}$ optical transitions in 209 and 210 francium isotopes
}

\author{
S. Agustsson ${ }^{1}$, G. Bianchi ${ }^{1}$, R. Calabrese ${ }^{2}$, L. Corradi ${ }^{3}$, A. Dainelli ${ }^{3}$, A. Khanbekyan ${ }^{1,2}$, C. \\ MARINELLI $^{1}$, E. MARIOTTI ${ }^{1, *}$, L. MARMUGI ${ }^{4}$, G. MAZZOCCA ${ }^{2}$, L. MOI ${ }^{1}$, L. RICCI ${ }^{5}$, L. STIACCINI ${ }^{1}$, AND \\ L. TOMASSETTI ${ }^{6}$ \\ ${ }^{1}$ DSFTA and CNISM, University of Siena, via Roma 56, 53100 Siena, Italy \\ ${ }^{2}$ Department of Physics and Earth Science, University of Ferrara and INFN, via Saragat 1, 44122 Ferrara, Italy \\ ${ }^{3}$ INFN - Laboratori Nazionali di Legnaro, viale dell'Università 2, 35020 Legnaro (PD), Italy \\ ${ }^{4}$ Department of Physics and Astronomy, University College London, Gower Street, London WC1E 6BT, UK \\ ${ }^{5}$ Physics Department, University of Trento, via Sommarive 14, 38123 Trento, Italy \\ ${ }^{6}$ Department of Mathematics and Computer Sciences, University of Ferrara and INFN, via Saragat 1, 44122 Ferrara, Italy \\ *Corresponding author: emilio.mariotti@unisi.it
}

Compiled September 14, 2017

We report on the direct experimental observation of the $7 \mathrm{p}^{2} \mathbf{P}_{3 / 2} \rightarrow 7 \mathrm{~d}^{2} \mathrm{D}$ optical transitions in 209 and 210 francium isotopes. By continuously monitoring the fluorescence emitted by the isotopes collected in a magnetooptical trap, the electric dipole transitions $7 \mathbf{p}^{2} \mathbf{P}_{3 / 2} \rightarrow$ $7 \mathrm{~d}^{2} \mathrm{D}_{5 / 2}$ of ${ }^{209} \mathrm{Fr}$, not yet experimentally observed, and $7 \mathbf{p}^{2} \mathbf{P}_{3 / 2} \rightarrow 7 d^{2} D_{5 / 2}, 7 \mathbf{p}^{2} \mathbf{P}_{3 / 2} \rightarrow 7 d^{2} D_{3 / 2}$ of ${ }^{210}$ Fr were detected as sub-Doppler depletion dips of the cold atom population. This approach allowed unambiguous identification of the excited state hyperfine structures, even in absence of a large stable vapor. Our findings demonstrate the effectiveness and the flexibility of fluorescence monitoring of trap depletion upon laser excitation, and broaden the experimental knowledge of francium isotopes and their electronic and nuclear properties. These results will have a relevant impact in ongoing researches for low-energy testing of fundamental symmetries with francium, from atomic parity nonconservation to the electron dipole moment.

OCIS codes: $\quad 300.6210,300.6320,020.3260,020.3320$

This is a preprint version of the article appeared in: Opt. Lett. 42, 18, 3682-3685 (2017) DOI: 10.1364/OL.42.003682. One print or electronic copy may be made for personal use only. Systematic reproduction and distribution, duplication of any material in this paper for a fee or for commercial purposes, or modifications of the content of this paper are prohibited.

Francium, the heaviest and rarest alkali, is a promising atom for probing fundamental symmetries and quantities [1], such as the measurement of the electric dipole moment of the electron [2]. A direct and detailed experimental knowledge of the atomic properties of Fr isotopes is a fundamental prerequisite for these investigations. Recent experimental data [3] prompted new theoretical studies of the Fr atomic structure $[4,5]$ and of the magnetic dipole and electric quadrupole hyperfine structure constants [6]. Fr, with its long chain of isotopes and simple electronics structure, has become an interesting candidate to tackle the challenge of atomic parity non-conservation (APNC) at low energy $[7,8]$, where the most precise experiments have been realized, albeit without any conclusive evidence of APNC [9], also because the lack of a sufficient precision of theoretical predictions for non-alkali atoms [10]. APNC effects are predicted to grow approximately as the third power of the atomic number $Z$ [11]. Therefore the use of an alkali atom and possibly heavier than Cs would allow for a more precise comparison with the Standard Model.

In this framework, laser spectroscopy of electronic states of the various isotopes is the main tool to investigate and experimentally confirm the fundamental properties of Fr nuclei, thanks to the extreme accuracy achievable in frequency measurements [12-14]. The isotopic shift $\Delta v_{A, A^{\prime}}$ of electronic transitions is directly related to variation in the nuclear mean square radius, $\left\langle r^{2}\right\rangle_{A, A^{\prime}}$. In addition, the electronic hyperfine structure is related to the nuclear spin, nuclear magnetic dipole and quadrupole momenta, and therefore also to the nuclear symmetry.

Francium has therefore been studied by laser spectroscopy [15-18] and in Magneto Optical Traps (MOTs) [3, 19, 20]. Nevertheless, francium energy structure is not yet adequately known, in particular with the level of accuracy required for APNC.

In this Letter, we report on the experimental detection of the francium $7 \mathrm{p}^{2} \mathrm{P}_{3 / 2} \rightarrow 7 \mathrm{~d}^{2} \mathrm{D}_{5 / 2}$ hyperfine optical transitions at $961 \mathrm{~nm}$ and the $7 \mathrm{p}^{2} \mathrm{P}_{3 / 2} \rightarrow 7 \mathrm{~d}^{2} \mathrm{D}_{3 / 2}$ hyperfine optical transitions at $969 \mathrm{~nm}$ (See Fig. 1). The fluorescence emitted by a MOT of the desired isotope is continuously monitored, while a probe laser is scanned in frequency across the expected resonance. On resonance, immediate MOT depletion is detected by a cooled CCD camera as a decrease of the fluorescence signal [21]. We have observed the $7 \mathrm{p}^{2} \mathrm{P}_{3 / 2} \rightarrow 7 \mathrm{D}$ optical transitions in both ${ }^{210} \mathrm{Fr}$ and ${ }^{209}$ Fr. For the latter, our data are the first reported in the scientific literature. 

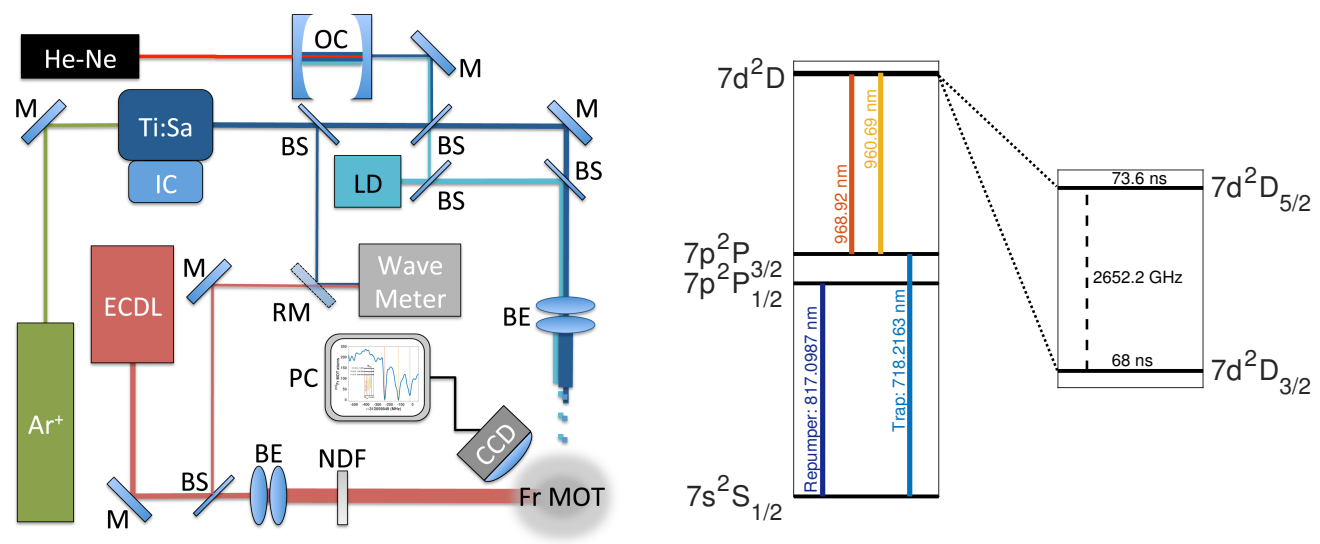

Fig. 1. Left: Low-energy section of the experimental setup. IC: intracavity reference. OC: optical cavity. LD: laser diode. ECDL: External Cavity Diode Laser. M: mirror; BS: beam-splitter; RM: removable mirror; BE: beam-expander; NDF: variable neutral density filter. Right: Fine structure of Fr levels of interest for the experiments and MOT optical transitions.

$\mathrm{Fr}^{+}$ions are produced by nuclear-evaporation in an ${ }^{197} \mathrm{Au}$ target hit by the INFN-LNL TANDEM XTU $100 \mathrm{MeV}{ }^{18} \mathrm{O}^{6+}$ beam at the WADE facility [22]. Ions are then extracted and routed by an electrostatic beam line to the MOT chamber, where they are neutralized inside a $25 \mu \mathrm{m}$ thick $\mathrm{Y}$ foil that acts as a local source of Fr atoms. The experimental chamber is a spherical Pyrex cell, with optical windows. Its inner surface is coated with poly-dimethylsiloxane to minimize atoms losses [23] and to ensure elastic atom/walls collisions. Pressure is $10^{-9} \mathrm{mbar}$. The MOT is in a 3D configuration, with a linear magnetic field gradient of $10 \mathrm{G} \mathrm{cm}^{-1}$. Trapping light at $718 \mathrm{~nm}$ is produced by a Ti:Sa laser pumped by an $\mathrm{Ar}^{+} 20 \mathrm{~W} \mathrm{CW}$ laser. The frequency of the Ti:Sa is locked to an intracavity reference and to an external optical cavity, stabilized with an ultra-stable He-Ne laser. The trap laser is red-detuned by about $4 \Gamma(\Gamma \approx 6 \mathrm{MHz})$. The total power is $350 \mathrm{~mW}$ for ${ }^{210} \mathrm{Fr}$ and $340 \mathrm{~mW}$ for ${ }^{209} \mathrm{Fr}$. A laser diode generates the repumping light at $817 \mathrm{~nm}$, locked to the stabilized optical cavity.

To achieve the flexibility required by different isotopes and the promptness of operation required by short lived atoms (the half-life is $50 \mathrm{~s}$ and $199 \mathrm{~s}$ for ${ }^{209} \mathrm{Fr}$ and ${ }^{210} \mathrm{Fr}$, respectively), detection of atomic transitions is performed by monitoring the change in fluorescence with the MOT depletion technique described above. In detail, the trap volume is constantly monitored by a cooled CCD camera. A real-time weighted background subtraction algorithm isolates light emitted by the atomic cloud and reduces the impact of lasers intensity noise. A narrowband interference filter eliminates spurious light, as well as scattered light from the excitation laser. In this way, detection is independent of the specific isotope in use $[19,21]$. The sensitivity of our detection system is $1 \mathrm{fW}$, corresponding to 10 atoms.

Optical transitions from the MOT excited state $\left(7 \mathrm{p}^{2} \mathrm{P}_{3 / 2}\right.$, $\mathrm{F}=15 / 2$ for ${ }^{210} \mathrm{Fr}$ and $7 \mathrm{p}^{2} \mathrm{P}_{3 / 2}, \mathrm{~F}=6$ for ${ }^{209} \mathrm{Fr}$, see Fig. 1) are excited by a probe laser (ECDL, [24]) that intercepts the cold Fr atoms and whose frequency is continuously scanned across the expected resonance (Fig. 1). When the excitation beam frequency matches that of an electronic hyperfine transition, Fr atoms are excited to a $7 d^{2} \mathrm{D}$ level and are thus forced out of the cooling cycling transition. This depletes the MOT population, whose minimum coincides with the center of the optical transition.

Observation in cold atoms has the obvious advantage of suppressing Doppler broadening. This approach allows frequency resolution of order $\Gamma$, without the need of conventional Dopplerfree techniques such as saturated absorption spectroscopy. It is worth noting that here, due to the absence of a stable and large vapor density of Fr, the latter would be unfeasible. By limiting the excitation beam intensity via a neutral density filter, the impact of power broadening is reduced. Nevertheless, laser-induced mechanisms such as Autler-Townes splitting can produce supplemental broadening of the transitions, as observed in $\mathrm{Rb}$ MOTs [21].

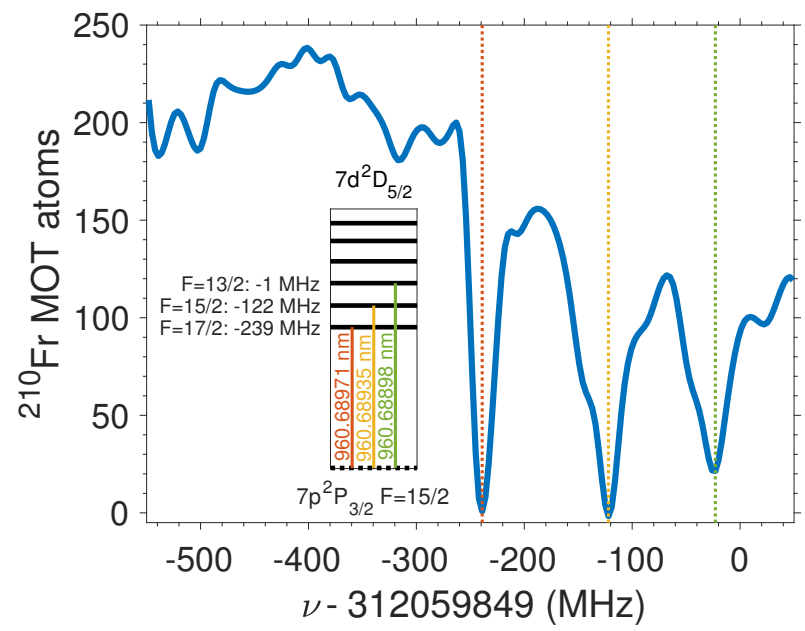

Fig. 2. ${ }^{210} \mathrm{Fr} 7 \mathrm{p}^{2} \mathrm{P}_{3 / 2}(\mathrm{~F}=15 / 2) \rightarrow 7 \mathrm{~d}^{2} \mathrm{D}_{5 / 2}\left(\mathrm{~F}^{\prime}=17 / 2,15 / 2\right.$, $13 / 2$ ) optical transitions observed as MOT population depletion caused by a laser beam of wavelength $960.7 \mathrm{~nm}$, intensity $1.98 \mathrm{~mW} \mathrm{~cm}^{-2}$. Inset: levels scheme according to [19, 25, 26].

In Fig. 2 we present results on the transitions $7 \mathrm{p}^{2} \mathrm{P}_{3 / 2}(\mathrm{~F}$ $=15 / 2) \rightarrow 7 \mathrm{~d}^{2} \mathrm{D}_{5 / 2}\left(\mathrm{~F}^{\prime}=17 / 2,15 / 2,13 / 2\right)$ in ${ }^{210} \mathrm{Fr}$. The inset shows the relevant hyperfine levels. Here and henceforth, unless otherwise stated, the detunings reported in the level schemes are based on measurements by the Stony Brook collaboration $[25,26]$ and on previous results by our group [19].

The excitation beam is generated by an ECDL (Sacher Lasertechnik Lion) tuned at $960.7 \mathrm{~nm}$ whose frequency is modulated over an interval of about $500 \mathrm{MHz}$ by a triangular waveform of period $140 \mathrm{~s}$ (sweep frequency of $7 \mathrm{mHz}$ ). Powers be- 
tween $1 \mathrm{~mW}$ and $15 \mathrm{~mW}$ were tested, with a $30 \mathrm{~mm}$ diameter beam spot at the trapping region. The laser frequency is continuously monitored by a fiber-coupled wavemeter (Burleigh $\mathrm{W}-1500)$, with a relative precision of $10^{-7}$. The calibration of the frequency axis of the experimental data relies on readings from the wavemeter. Accuracy was confirmed with independent measurements of known optical transitions, such as the trap and repumper ones.

When the ECDL is resonant with one of the hyperfine components of the $7 \mathrm{p}^{2} \mathrm{P}_{3 / 2} \rightarrow 7 \mathrm{~d}^{2} \mathrm{D}_{5 / 2}$ transition, ${ }^{210} \mathrm{Fr}$ atoms in the $7 \mathrm{p}^{2} \mathrm{P}_{3 / 2}(\mathrm{~F}=15 / 2)$ leave the MOT. This mechanism produces a rapid decrease of the population, as demonstrated by the three minima in Fig. 2.

When the ECDL is no longer resonant, trapping progressively regains its initial efficiency and the MOT is repopulated. Unlike the depletion process which is dominated by the timescale of photon/atom interaction, the return to equilibrium of the cold atoms population involves also the MOT loading time. Hence, it can be significantly slower than the previous one. To reduce such effect, the sweep frequency is set such that the interaction time is longer than the characteristic MOT loading time at any frequency. This solution ensures dynamical equilibrium during probing of the Fr energy levels (see Ref. [21]) and allows detection of the complete set of dipole-allowed transitions in ${ }^{210} \mathrm{Fr}: 7 \mathrm{p}^{2} \mathrm{P}_{3 / 2}(\mathrm{~F}$ $=15 / 2) \rightarrow 7 d^{2} D_{5 / 2}\left(F^{\prime}=17 / 2,15 / 2,13 / 2\right)$. When the probe laser intensity is optimized for the largest contrast in presence of atomic transitions, complete depletion of the MOT population is observed at resonance because of direct absorption of a probe photon. The experimental uncertainty is $\pm 10 \mathrm{MHz}$, as given by the wavemeter readout.

The measured frequencies of the $\mathrm{F}^{\prime}=17 / 2$ and the $\mathrm{F}^{\prime}=15 / 2$ are in very good agreement with previous experimental data $[25,26]$. The third level, $\mathrm{F}^{\prime}=13 / 2$, is slightly shifted to a lower frequency with respect to the expected value: the experimental detuning is $(-20 \pm 10) \mathrm{MHz}$, while the reference value is $(0 \pm 5) \mathrm{MHz}$, as calculated from $A_{h f s}^{(210)}=(-17.8 \pm 0.8) \mathrm{MHz}$ and $B_{h f s}^{(210)}=(64 \pm 17) \mathrm{MHz}$ [25]. The observed FWHMs are up to 5 times larger than the predicted natural linewidth due to the $7 \mathrm{p}^{2} \mathrm{P}_{3 / 2}$ level, despite the relatively low intensity of the excitation laser $\left(\leq 2.1 \mathrm{~mW} \mathrm{~cm}^{-2}\right)$. Incidentally, we recall here that the natural linewidth of the $7 \mathrm{~d}^{2} \mathrm{D}_{5 / 2}$ is around $2 \mathrm{MHz}$. Although residual power broadening cannot be neglected, the main broadening mechanism is Autler-Townes splitting, induced by the trap laser on the $7 \mathrm{p}^{2} \mathrm{P}_{3 / 2}$ $(\mathrm{F}=15 / 2)$ level, which acts as a ground state for the transitions under investigation. The intense trap beams cause a powerinduced splitting of the cooling excited state, thus broadening the resonant band for the $7 d^{2} D_{5 / 2}$ transitions. Consequently, the absorption cross-section for the excitation laser is broader than the natural linewidth [21]. At the current stage of investigation, one may argue that Autler-Townes plays a role also in the asymmetry of the peaks and in the related frequency shift, as noted in [21]. This could account for the shape and frequency shift of the observed peaks.

The experiment is repeated for the ${ }^{210} \mathrm{Fr}$ $7 \mathrm{p}^{2} \mathrm{P}_{3 / 2} \quad(\mathrm{~F}=15 / 2) \rightarrow 7 \mathrm{~d}^{2} \mathrm{D}_{3 / 2} \quad\left(\mathrm{~F}^{\prime}=13 / 2,15 / 2\right)$ optical transitions, with the ECDL tuned at $968.9 \mathrm{~nm}$. Results are shown in Fig. 3.

In this case, only two hyperfine components are allowed for an electric dipole transition. Accordingly, only two minima, marking the position of the two most energetic levels of the $7 d^{2} D_{3 / 2}$ manifold, are visible in Fig. 3. The resonant frequencies are in very good agreement with the expected values reported

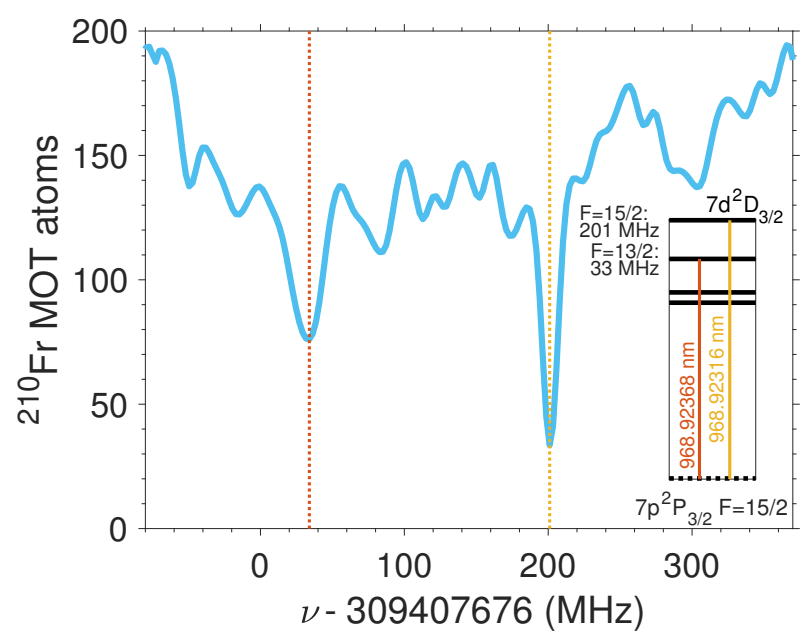

Fig. 3. ${ }^{210} \mathrm{Fr} 7 \mathrm{p}^{2} \mathrm{P}_{3 / 2}(\mathrm{~F}=15 / 2) \rightarrow 7 \mathrm{~d}^{2} \mathrm{D}_{3 / 2}\left(\mathrm{~F}^{\prime}=13 / 2,15 / 2\right)$ optical transitions observed as MOT population depletion caused by a laser beam of wavelength $968.9 \mathrm{~nm}$, intensity $1.77 \mathrm{~mW} \mathrm{~cm}^{-2}$. Inset: levels scheme according to [19, 25, 26].

in the inset. Broadening and distortions are less evident than in Fig. 2 because of the larger separation of the two transitions, which implies a smaller impact of the Autler-Townes splitting of the $7 \mathrm{p}^{2} \mathrm{P}_{3 / 2}(\mathrm{~F}=15 / 2)$ level. However, a small residual asymmetry of the $\mathrm{F}^{\prime}=13 / 2$ peak is present.

We show in Fig. 4 the $7 p^{2} P_{3 / 2}(F=6) \rightarrow 7 d^{2} D_{5 / 2}\left(F^{\prime}=7,6\right.$, 5) transition for ${ }^{209} \mathrm{Fr}$, which has never been directly detected before. Albeit the number of atoms is lower than in the previous case - because of the smaller yield of our nuclear-evaporation reaction for this isotope [27] - the three electric dipole transitions dips are clearly observed, with almost $100 \%$ contrast in each case.

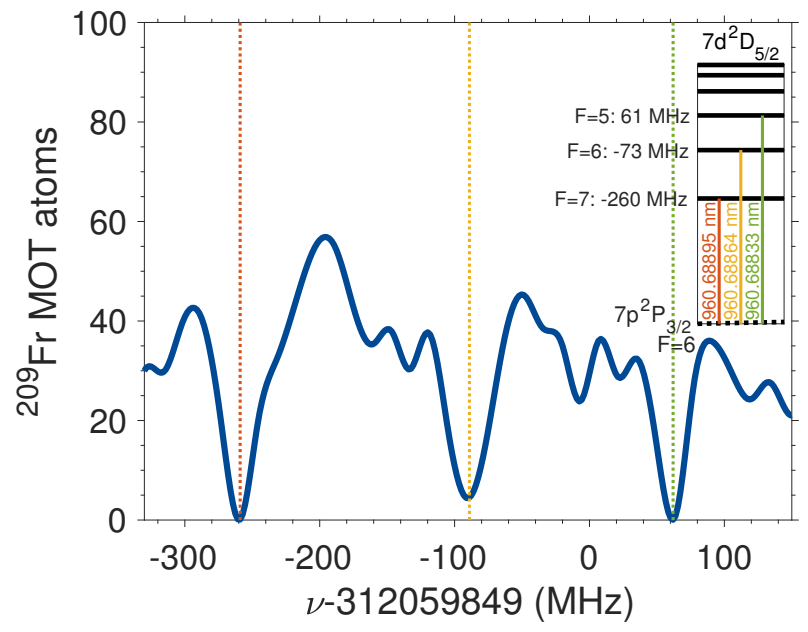

Fig. 4. ${ }^{209} \mathrm{Fr} 7 \mathrm{p}^{2} \mathrm{P}_{3 / 2}(\mathrm{~F}=6) \rightarrow 7 \mathrm{~d}^{2} \mathrm{D}_{5 / 2}\left(\mathrm{~F}^{\prime}=7,6,5\right)$ optical transitions observed as MOT population depletion caused by a laser beam $960.7 \mathrm{~nm}$, intensity $2.1 \mathrm{~mW} \mathrm{~cm}^{-2}$. Inset: levels scheme according to Eq. 1.

The expected relative positions of the hyperfine transitions of ${ }^{209} \mathrm{Fr}$, in absence of other data in literature, were calculated from the hyperfine splitting formula: 


$$
\Delta E_{h f_{s}} \simeq \frac{1}{2} A_{h f_{s}} K+B_{h f_{s}} \frac{\frac{3}{2} K(K+1)-2 I(I+1) J(J+1)}{2 I(2 I-1) 2 J(2 J-1)} .
$$

Here, $K=F(F+1)-I(I+1)-J(J+1), I$ is the nuclear spin, $J$ and $F$ are the electronic and atomic angular momentum number, respectively. The hyperfine splitting coefficients $A_{h f s}$ and $B_{h f_{s}}$ were calculated by re-scaling the ${ }^{210} \mathrm{Fr}$ experimental values reported in [25] for the 209 isotope [28], using the general relations of Ref. [12]. Following this procedure, we have obtained, in the case of ${ }^{209} \mathrm{Fr}, A_{h f s}^{(209)}=(-21 \pm 1) \mathrm{MHz}$ and $B_{h f s}^{(209)}=(-81 \pm 22) \mathrm{MHz}$. The uncertainties are calculated from the experimental values of [25].

Two of the three minima in Fig. 4 are in excellent agreement with the estimated frequencies and hence with the corresponding hyperfine splittings (see Tab. 1). Broadening due to AutlerTownes splitting of the $7 \mathrm{p}^{2} \mathrm{P}_{3 / 2}\left(\mathrm{~F}^{\prime}=6\right)$ level is observed, as well as the related small deformation and detuning of the resonant peaks. We note however that even in this case experimental values and predictions are consistent.

\begin{tabular}{ccc}
\hline Excited Level & Experiment (This work) & Theory (Eq. 1) \\
\hline $\mathrm{F}^{\prime}=7$ & $(-259 \pm 10) \mathrm{MHz}$ & $(-260 \pm 15) \mathrm{MHz}$ \\
$\mathrm{F}^{\prime}=6$ & $(-89 \pm 10) \mathrm{MHz}$ & $(-73 \pm 7) \mathrm{MHz}$ \\
$\mathrm{F}^{\prime}=5$ & $(+62 \pm 10) \mathrm{MHz}$ & $(+61 \pm 7) \mathrm{MHz}$ \\
\hline
\end{tabular}

Table 1. ${ }^{209} \mathrm{Fr} 7 \mathrm{p}^{2} \mathrm{P}_{3 / 2} \rightarrow 7 \mathrm{~d}^{2} \mathrm{D}_{5 / 2}$ hyperfine splittings.

In conclusion, we have directly observed the optical transitions $7 \mathrm{p}^{2} \mathrm{P}_{3 / 2} \rightarrow 7 \mathrm{D}_{3 / 2}, 7 \mathrm{~d}^{2} \mathrm{D}_{5 / 2}$ in a MOT of ${ }^{210} \mathrm{Fr}$ and, for the first time, the optical transitions $7 \mathrm{p}^{2} \mathrm{P}_{3 / 2} \rightarrow 7 \mathrm{~d}^{2} \mathrm{D}_{5 / 2}$ in a MOT of ${ }^{209} \mathrm{Fr}$. Detection was possible by MOT depletion in presence of an excitation laser. By taking advantage of the low temperature of the MOTs, we have observed in details - for the two isotopes the hyperfine structure of the $7 \mathrm{~d}^{2} \mathrm{D}$ manifold and determined the energy levels, with very good agreement with available data and theoretical predictions.

Our results not only validate the MOT depletion for highresolution spectroscopy of unknown and unstable species, but also increase the experimental knowledge of Fr isotopes, which is a paramount prerequisite for advanced measurements such as the electron dipole moment. Finally, we note that our results represent a preliminary test of our experimental protocol on an electric dipole transition, in view of the investigation of the electric quadrupole $7 \mathrm{~s}^{2} \mathrm{~S}_{1 / 2} \rightarrow 6 \mathrm{~d}^{2} \mathrm{D}$ transitions, not yet observed but of major interest for APNC.

This work has been funded by INFN-CSN5.

WADE is grateful to the technical staff of Universities of Ferrara and Siena and of INFN-LNL. The authors gratefully acknowledge the support of the TANDEM XTU operators.

\section{REFERENCES}

1. J. Ginges and V. Flambaum, Physics Reports 397, 63 (2004).

2. Y. Sakemi, K. Harada, T. Hayamizu, M. Itoh, H. Kawamura, S. Liu, H. S. Nataraj, A. Oikawa, M. Saito, T. Sato, H. P. Yoshida, T. Aoki, A. Hatakeyama, T. Murakami, K. Imai, K. Hatanaka, T. Wakasa, Y. Shimizu, and M. Uchida, Journal of Physics: Conference Series 302, 012051 (2011).
3. E. Gomez, L. A. Orozco, and G. D. Sprouse, Reports on Progress in Physics 69, 79 (2006).

4. U. I. Safronova, W. R. Johnson, and M. S. Safronova, Phys. Rev. A 76, 042504 (2007).

5. B. K. Sahoo, D. K. Nandy, B. P. Das, and Y. Sakemi, Phys. Rev. A 91, 042507 (2015).

6. S. Singh, B. K. Sahoo, and B. Arora, Phys. Rev. A 94, 023418 (2016).

7. E. Gomez, S. Aubin, R. Collister, J. A. Behr, G. Gwinner, L. A. Orozco, M. R. Pearson, M. Tandecki, D. Sheng, and J. Zhang, Journal of Physics: Conference Series 387, 012004 (2012).

8. B. K. Sahoo, T. Aoki, B. P. Das, and Y. Sakemi, Phys. Rev. A 93, 032520 (2016).

9. C. S. Wood, S. C. Bennett, D. Cho, B. P. Masterson, J. L. Roberts, C. E. Tanner, and C. E. Wieman, Science 275, 1759 (1997).

10. K. Tsigutkin, D. Dounas-Frazer, A. Family, J. E. Stalnaker, V. V. Yashchuk, and D. Budker, Phys. Rev. Lett. 103, 071601 (2009).

11. M.-A. Bouchiat and C. Bouchiat, Reports on Progress in Physics 60, 1351 (1997).

12. A. Pálffy, Contemporary Physics 51, 471 (2010).

13. H.-J. Kluge and W. Nörtershäuser, Spectrochimica Acta Part B: Atomic Spectroscopy 58, 1031 (2003).

14. J. Billowes and P. Campbell, Journal of Physics G: Nuclear and Particle Physics 21, 707 (1995).

15. S. Liberman, J. Pinard, H. T. Duong, P. Juncar, P. Pillet, J.-L. Vialle, P. Jacquinot, F. Touchard, S. Büttgenbach, C. Thibault, M. de SaintSimon, R. Klapisch, A. Pesnelle, and G. Huber, Phys. Rev. A 22, 2732 (1980).

16. A. Coc, C. Thibault, F. Touchard, H. Duong, P. Juncar, S. Liberman, J. Pinard, J. Lermé, J. Vialle, S. Büttgenbach, A. Mueller, and A. Pesnelle, Physics Letters B 163, 66 (1985).

17. E. Arnold, W. Borchers, H. T. Duong, P. Juncar, J. Lerme, P. Lievens, W. Neu, R. Neugart, M. Pellarin, J. Pinard, J. L. Vialle, K. Wendt, and ISOLDE, Journal of Physics B: Atomic, Molecular and Optical Physics 23, 3511 (1990).

18. R. P. de Groote, I. Budinčević, J. Billowes, M. L. Bissell, T. E. Cocolios, G. J. Farooq-Smith, V. N. Fedosseev, K. T. Flanagan, S. Franchoo, R. F. Garcia Ruiz, H. Heylen, R. Li, K. M. Lynch, B. A. Marsh, G. Neyens, R. E. Rossel, S. Rothe, H. H. Stroke, K. D. A. Wendt, S. G. Wilkins, and X. Yang, Phys. Rev. Lett. 115, 132501 (2015).

19. S. Sanguinetti, R. Calabrese, L. Corradi, A. Dainelli, A. Khanbekyan, E. Mariotti, C. de Mauro, P. Minguzzi, L. Moi, G. Stancari, L. Tomassetti, and S. Veronesi, Opt. Lett. 34, 893 (2009).

20. J. Zhang, M. Tandecki, R. Collister, S. Aubin, J. A. Behr, E. Gomez, G. Gwinner, L. A. Orozco, M. R. Pearson, and G. D. Sprouse, Phys. Rev. Lett. 115, 042501 (2015).

21. L. Moi, G. Batignani, A. Khanbekyan, K. Khanbekyan, C. Marinelli, E. Mariotti, L. Marmugi, L. Corradi, A. Dainelli, R. Calabrese, G. Mazzocca, L. Tomassetti, and P. Minguzzi, Measurement Science and Technology 24, 015201 (2013).

22. E. Mariotti, A. Khanbekyan, C. Marinelli, L. Marmugi, L. Moi, L. Corradi, A. Dainelli, R. Calabrese, G. Mazzocca, and L. Tomassetti, International Journal of Modern Physics E 23, 1430009 (2014).

23. S. Agustsson, G. Bianchi, R. Calabrese, L. Corradi, A. Dainelli, A. Khanbekyan, C. Marinelli, E. Mariotti, L. Marmugi, L. Ricci, L. Stiaccini, L. Tomassetti, and A. Vanella, Scientific Reports 7, 4207 (2017).

24. L. Ricci, M. Weidemüller, T. Esslinger, A. Hemmerich, C. Zimmermann, V. Vuletic, W. König, and T. Hänsch, Optics Communications 117, 541 (1995).

25. J. M. Grossman, R. P. Fliller, T. E. Mehlstäubler, L. A. Orozco, M. R. Pearson, G. D. Sprouse, and W. Z. Zhao, Phys. Rev. A 62, 052507 (2000).

26. J. M. Grossman, R. P. Fliller, L. A. Orozco, M. R. Pearson, and G. D. Sprouse, Phys. Rev. A 62, 062502 (2000).

27. L. Corradi, B. R. Behera, E. Fioretto, A. Gadea, A. Latina, A. M. Stefanini, S. Szilner, M. Trotta, Y. Wu, S. Beghini, G. Montagnoli, F. Scarlassara, R. N. Sagaidak, S. N. Atutov, B. Mai, G. Stancari, L. Tomassetti, E. Mariotti, A. Khanbekyan, and S. Veronesi, Phys. Rev. C 71, 014609 (2005). 
28. K. M. Lynch, J. Billowes, M. L. Bissell, I. Budinčević, T. E. Cocolios, R. P. De Groote, S. De Schepper, V. N. Fedosseev, K. T. Flanagan, S. Franchoo, R. F. Garcia Ruiz, H. Heylen, B. A. Marsh, G. Neyens, T. J. Procter, R. E. Rossel, S. Rothe, I. Strashnov, H. H. Stroke, and K. D. A. Wendt, Phys. Rev. X 4, 011055 (2014). 